\title{
Extremely low frequency magnetic field exposure affects DnaK and GroEL expression in $E$. coli cells with impaired heat shock response
}

\author{
Brunella Del Re ${ }^{1,3}$, Pamela Marcantonio ${ }^{1}$, Ferdinando Bersani ${ }^{2,3,4}$ and Gianfranco Giorgi ${ }^{1,3}$ \\ ${ }^{1}$ Department of Evolutionary Experimental Biology, University of Bologna, via Selmi 3, 40126 Bologna, Italy \\ 2 Department of Physics, University of Bologna, via Berti Bichat 6/2, 40127 Bologna, Italy \\ ${ }^{3}$ Inter-departmental Center "L. Galvani", via San Giacomo 12, 40126 Bologna, Italy \\ ${ }^{4}$ Consorzio Interuniversitario Istituto Nazionale Biostrutture e Biosistemi, viale Medaglie d'oro 305, 00136 Roma, Italy
}

\begin{abstract}
In our earlier experiments, we found that extremely low frequency magnetic fields (ELF$\mathrm{MF}$ ) affect heat shock protein (HSP) expression in wild type Escherichia coli cells. In the present work we investigate the ability of ELF-MF exposure to trigger an increase of DnaK and GroEL protein levels also in E. coli cells not exhibiting the classic heat shock response (HSR) when subjected to a $42^{\circ} \mathrm{C}$ heat stress. We find that these cells, although lacking a HSR to heat shock treatment, show an enhancement of DnaK and GroEL protein levels after 30 or 90 min sinusoidal ELF-MF exposure $(50 \mathrm{~Hz}, 1 \mathrm{mT})$. This result suggests that the HSP induction pathway triggered by ELF-MF exposure could be different from that elicited by heat shock treatment.
\end{abstract}

Key words: Escherichia coli - Heat shock protein - Transposition - Extremely low frequency magnetic field - Sinusoidal magnetic field

Abbreviations: ELF-MF, extremely low frequency magnetic field; HSP, heat shock protein; HSR, heat shock response; Tr, transformed cells (with high transposition activity); NTr, non transformed cells (without high transposition activity).

In recent years there has been an increasing interest in the investigation on biological effects of magnetic field (MF) mainly because of the frequent use of electric devices in everyday activities by people of developed countries. Many studies on extremely low frequency (ELF)-MF have showed that exposure to these fields can affect biological functions such as RNA transcription, cell viability, cell proliferation although it is unknown which molecular mechanisms are involved (Babincova et al. 2000; Ventura et al. 2005; Williams et al. 2006; Kroupova et al. 2007; Novak et al. 2007; Cellini et al. 2008). Still it is not still clear whether ELF-MF exposure can represent a stress factor for the cells.

In order to clarify this topic, many researchers carried out experiments to verify whether ELF-MF exposure could induce a "heat shock response" (HSR) that is often involved in the cell response to stress. Actually HSR was initially

Correspondence to: Gianfranco Giorgi, Dip. di Biologia Evoluzionistica Sperimentale, Via Selmi 3, 40126 Bologna, Italy E-mail: gianfranco.giorgi@unibo.it discovered as a cellular response to heat shock but more recently literature has emerged that offers findings about HSR induction by other stresses such as DNA damage, oxidative stress, ethanol, $\mathrm{CdCl}$, antibiotics, heavy metal, phage infection, carbon source or amino acid starvation (Yura et al. 2000).

HSR is characterized by increased synthesis of a set of protein (heat stock protein - HSP) that function by facilitating the refolding of damaged proteins or by eliminating proteins that cannot be repaired. Among the HSPs the most highly stress induced are the $70 \mathrm{kDa}$ protein (HSP70) and the 60 $\mathrm{kDa}$ protein (HSP60), present in almost all the organisms from E. coli (DnaK and GroEL, respectively) to humans.

Some authors reported a HSP induction by ELF-MF exposure (Smith 1996; Goodman and Blank M 1998; Chow and Tung 2000; Gutzeit 2001; Del Re et al. 2006) whereas others did not find it (Nakasono and Saiki 2000; Henderson et al. 2003; Bernardini et al. 2007); however, it is important to point out that results obtained by different authors are not always comparable being based on different experimental 
designs and models. In synthesis, since experimental data about this topic are rather controversial, there is no general agreement about the capability of ELF-MF to affect HSP expression. It has been suggested that MFs, differently from other stress agents, do not lead to reproducible induction of HSPs but elicit a moderate variation of the biological function depending on the global physiology of the cell (Coult et al. 2004; Henderson et al. 2006). However, some potential biomedical applications of electromagnetic field induction of HSPs have been described: George et al. (2008) have reported that myocardial function, following ischemia, can be significantly improved by preventive up-regulation of the HSP70 gene induced by exposure to $60 \mathrm{~Hz}$ electromagnetic field. Therefore these observations urge further investigation of MF effects on HSP gene expression.

In a previous paper we carried out a comparative analysis on the HSP induction in E. coli cells by the exposure to two different MF signals and we found that ELF-MF can affect expression of HSP $70 \mathrm{kDa}$ protein (DnaK) and of HSP 60 $\mathrm{kDa}$ protein (GroEL) depending on the wave shape (Del Re et al. 2006).

In the present study our aim was to investigate the ability of ELF-MF to induce HSP in cells showing an impaired HSR to heat stress $\left(42^{\circ} \mathrm{C}\right)$ in order to verify if the two response pathways overlap.

Experiments were performed using the following E. coli bacterial strain: DH5a [F-, $\varphi 80$ dlacZ $\Delta \mathrm{M} 15$, endA1, recA1, hsdR17 $\left[\mathrm{r}_{\mathrm{k}}{ }^{-}, \mathrm{m}_{\mathrm{k}}{ }^{+}\right]$, supE44, thi-1, gyrA96, relA1, $\Delta$ [lacZYA$\left.\operatorname{argF}] \mathrm{U} 169, \lambda^{-}\right]$.

Moreover two plasmids were used: pNK1207 [ATCC] and pNK2882 [ATCC]. pNK1207 is a pBR322 derivative that carries a selectable $\mathrm{Amp}^{\mathrm{r}}$ marker, and contains the mini-Tn10 transposon without transposase gene. pNK2882, a pACYC184 derived, carries a selectable $\operatorname{Tet}^{\mathrm{r}}$ marker and is compatible with pBR322-derived plasmid; the plasmid contains the ats 1 and ats 2 transposase gene that permits relaxed insertion specificity (Huisman and Kleckner 1987).

DH5a was not transformed (indicated as NTr) or was transformed (indicated as Tr) with both pNK1207 and pNK2882 plasmids. In NTr cells there is not transposition activity whereas in Tr cells the transposition frequency of the mini-Tn10 transposon is very high (Huisman and Kleckner 1987). NTr cells were grown in Luria Bertani (LB) medium; $\mathrm{Tr}$ cells were cultured in LB supplemented with ampicillin $(25 \mu \mathrm{g} / \mathrm{ml})$ and tetracycline $(50 \mu \mathrm{g} / \mathrm{ml})$.

Tr and NTr cells were used in our earlier experiments dealing Tn10 mobility (Del Re et al. 2003, 2004). In the present study we evaluated their HSR to heat stress and to ELF-MF exposure using the following experiment scheme. Briefly, saturated cultures were diluted $1: 100$ into LB medium, supplemented with the antibiotic if required, and incubated at $30^{\circ} \mathrm{C}$ until the cells reached the logarithmic phase $(0.22$ O.D.). Then the culture was divided into aliquots that were incubated for 30 or $90 \mathrm{~min}$ in round shaped tubes (diameter $16 \mathrm{~mm}$, Sterilin bijou) without agitation at the following conditions: i) $30^{\circ} \mathrm{C}$; ii) $42^{\circ} \mathrm{C}$ (heat shock); iii) $30^{\circ} \mathrm{C}$ plus ELF-MF exposure; iv) $30^{\circ} \mathrm{C}$ plus sham exposure.

The exposure system consisted of two pairs of Helmholtz coils, $23 \mathrm{~cm}$ diameter, $40(20+20)$ turns, which were doublewrapped in order to obtain wound (active coil) or counterwound configuration. In the counter-wound configuration, the current is the same as in the active coil but the MF is zero (sham). The coils were powered by a home-made DC current amplifier connected to a signal generator (Beckman FG3A). A MF signal of $50 \mathrm{~Hz}$ sinusoidal was used and intensity (rms) was $1 \mathrm{mT}$. The MF was measured by means of a Bell gaussmeter (model 7010); the error in the magnetic flux density $(\mathrm{dB} / \mathrm{dt})$ values was of the order of $2 \%$. The maximum $\mathrm{dB} / \mathrm{dt}$ at $1 \mathrm{mT}$ was about $0.14 \mathrm{~T} / \mathrm{s}$. This parameter is important, being directly related to the induced electric field within the cells and/or the Petri dish. Both the active and the sham coils were maintained in a thermostatic room at a constant temperature of $30^{\circ} \mathrm{C}$. The background field within the incubator was also measured: the static component (local geomagnetic field) was $50 \mu \mathrm{T}$ and the alternating current (A.C.) component was of the order of $0.3 \mu \mathrm{T}$, as measured by a sensitive probe (EMDEX II, Enertech). The experiments were conducted in blind. The tubes were placed in the centre of the coil system where the field uniformity was within $1 \%$.

After exposure cells were harvested and the total protein was extracted. Each experiment was performed in quadruplicate. Samples containing $10 \mu \mathrm{g}$ of total protein, measured by Bradford assay (Biorad Laboratories), were subjected to electrophoresis in a precast polyacrylamide gel (10\% BisTris XT-Criterion, Biorad) and then electro-transferred to polyvinylidene fluoride (pvdf) membranes (Amersham). For immunoblotting membranes were probed with monoclonal mouse IgG anti-DnaK, anti-GroEL (StressGen Biotechnologies Corp.), IgG anti-RNA polymerase a subunit (an house-keeping gene product used as control) (NeoClone Biotechnology International) as primary antibodies. The immunoreactive proteins were detected using alkaline phosphatase/conjugated anti-mouse rabbit IgG (StressGen Biotechnologies Corp.) then stained with BCIP (5-bromo4-chloro-3'-indolyphosphate p-toluidine salt) and NBT (nitro-blue tetrazolium chloride) (Biorad). Immunoblots were scanned and the optical density (OD) of each band was determined using a densitometer (Media Cybernetics, Image-Pro Plus Family imaging software, MD, USA).

To normalize OD values of HSP bands based on the amount of proteins loaded in each well, we took the average OD of all RNA polymerase a subunit bands and then calculated the normalizing factor for each lane by dividing the OD of each individual RNA polymerase $\alpha$ subunit band by the average OD of all RNA polymerase $\alpha$ subunit bands. The normalized OD of each HSP band was obtained by 


\section{HS treatment}

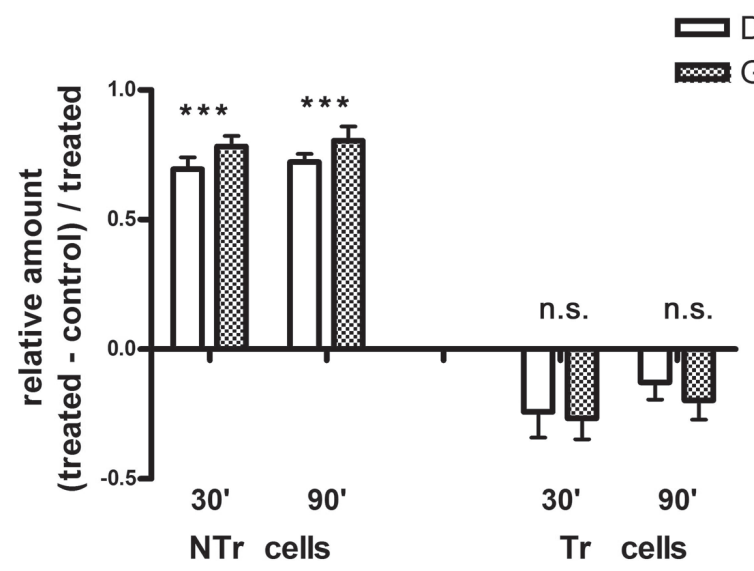

Figure 1. Comparison of DnaK and GroEL protein levels after subjecting NTr and $\operatorname{Tr}$ cells to heat shock (HS) $\left(42^{\circ} \mathrm{C}\right)$ for different times (30 or $90 \mathrm{~min}$ ). Data are expressed as: (treated - control)/ treated. The results of six independent experiments are combined. ${ }^{* * *} p<0.001$; n.s., not significant.

dividing the non-normalized OD of the HSP band by the normalizing factor. Finally, the average of the normalized OD of four HSP bands of each treatment was taken and compared.

Figure 1 shows DnaK and GroEL protein levels of Tr and NTr cells after heat shock treatment. Data are presented as relative quantities: exposed minus non-exposed divided by major value. Due to this intrinsic normalization data are directly comparable irrespective of plus or minus sign. Student's $t$-test was used for statistical analysis and $p$ values less than 0.05 were considered significant.

In NTr cells, DnaK and GroEL protein levels significantly increased after $30 \mathrm{~min}$ and even more significantly after 90 min of treatment $\left(42^{\circ} \mathrm{C}\right)$ as compared with controls $\left(30^{\circ} \mathrm{C}\right)$. On the contrary unexpectedly in Tr cells, HSP levels did not enhance but lightly decrease both after $30 \mathrm{~min}$ and $90 \mathrm{~min}$ as compared with controls, showing clearly an impaired HSR ability. Therefore Tr cells, compared to NTr cells, seemed to us to be a good model to gain information about overlapping between HSP induction pathway triggered by heat stress and that elicited by ELF-MF exposure. Then we exposed both $\mathrm{Tr}$ and NTr cells to ELF-MF or to sham for 30 or 90 min and after the exposure DnaK and GroEL protein levels were evaluated by Western blot.

Figure 2 shows the obtained results. An increase in DnaK and GroEL in both cell types was found as compared with sham-exposed controls. All the enhancements were significant with respect to the sham-exposed control except for $\mathrm{Tr}$ cells after $90 \mathrm{~min}$ of exposure. In all experiments no significant difference was observed neither between proteins

\section{MF treatment}

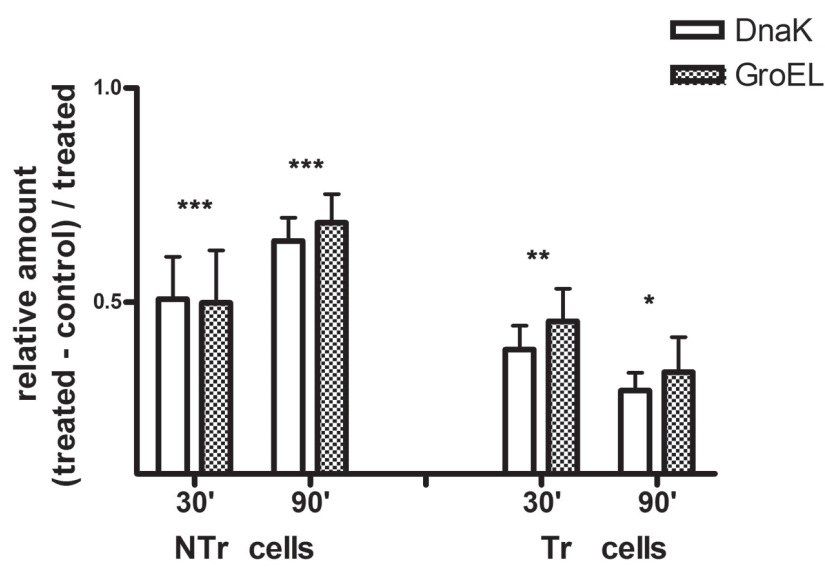

Figure 2. Comparison of DnaK and GroEL protein levels after subjecting NTr cells and Tr cells to sinusoidal magnetic field (SMF) $\left(1 \mathrm{mT}, 50 \mathrm{~Hz}\right.$, at $\left.30^{\circ} \mathrm{C}\right)$, for different times $(30$ or $90 \mathrm{~min})$. Data are expressed as: (treated - control)/treated. The results of six independent experiments are combined. ${ }^{* *} p<0.001 ;{ }^{* *} p<0.01 ;{ }^{*} p<0.05$.

(DnaK and GroEL) nor between exposure times (30 and 90) evaluated by a multifactor Anova test.

Bacteria have developed several complex mechanisms to cope with environmental modifications. It is well known that each stress condition elicits a specific cellular response: a SOS response is triggered by a DNA damage, a starvation response is elicited by a nutrient limit condition, HSR is induced by a temperature up shift and so on.

By using a specific cellular response the bacteria cell tries to optimize the synthesis of a specific set of proteins able to avoid any alteration in the normal metabolism due to an environmental modification. However, a single stimulus can induce more than one response and the same response can be induced by different response pathways.

ELF-MF exposure has been reported to induce an increased HSP expression (Smith 1996; Goodman and Blank 1998; Chow et al. 2000; Coult et al. 2004; Del Re et al. 2006) but the molecular pathways involved have not yet been investigated.

We have evaluated DnaK and GroEL expression after ELFMF exposure in E. coli $\mathrm{Tr}$ cells, with an impaired response to heat shock, as well as in E. coli NTr cells, showing a regular HSR ability. We found that ELF-MF exposure elicits an increased DnaK and GroEL expression in both $\mathrm{NTr}$ and $\mathrm{Tr}$ cells. Therefore HSP induction pathway triggered by ELF-MF seems not to be affected by the lack of functionality of HSR pathway elicited by heat stress. Tr cells show a high transposition activity that can have dramatic effects on the host cell. Our data suggest that some factors involved in transposition control could interfere somehow on the HSP induction by 
heat shock but not on that by ELF-MF. Work is in progress to understand the molecular mechanisms that underlay the observed impaired HSR in Tr cells.

HSP induction in E. coli is regulated by a very complex network which is currently not completely understood (Guisbert et al. 2008). At the level of transcription initiation, the recognition of heat shock promoters is mediated by the alternative sigma factor $\sigma^{32}$ that is tightly controlled mainly at the translational and posttranslational levels, where controlled proteolysis play a crucial role (Guisbert et al. 2004). It is well known that proteases are involved in remodeling the bacterial proteome in response to changes in cellular environment; since they play a role both in HSR and in transposition regulation (Twiss et al. 2005) they could be involved in the observed phenomena.

Our results seem to suggest that heat shock and ELFMF could elicit different response pathways. It has been hypothesized that ELF-MFs interfere with chemical reactions involving free radical production (Lacy-Hulbert et al. 1998; Di Carlo et al. 2001; Wolf et al. 2005); it could be possible that they induce also an oxidative stress response able to interact with HSPs regulation (Winter et al. 2005). Moreover it has been hypothesized that ELF-MFs are able to stimulate the transcription by interacting with electrons in DNA (Blank and Goodman 2008), in particular in eukaryotic cells, MF has been shown to cause an up-regulation of HSP70 by acting on a part of the promoter that is different from that stimulated by elevated temperatures. Our data seem to indicate such a phenomenon could also occur in bacterial cells.

In conclusion, with this study we provide evidence that 50 $\mathrm{Hz}$ MF can induce HSP expression in E. coli with impaired HSR to heat shock. In our opinion Tr cells could represent a good model for further studies aimed at the elucidation of the molecular mechanisms involved in the biological effects of MF exposure.

Acknowledgements. The work was partially supported by the PRIN 2005 MIUR ("Effects of electromagnetic fields on nervous cells").

\section{References}

Bernardini C., Zannoni A., Turba M. E., Bacci M. L., Forni M., Mesirca P., Remondini D., Castellani G., Bersani F. (2007): Effects of $50 \mathrm{~Hz}$ sinusoidal magnetic fields on Hsp27, Hsp70, Hsp90 expression in porcine aortic endothelial cells (PAEC). Bioelectromagnetics 28, 231-237; doi:10.1002/bem.20299

Babincová M., Sourivong P., Leszczynska D., Babinec P. (2000): Influence of alternating magnetic fields on two-dimensional tumor growth. Electromagn. Biol. Med. 19, 351-355; doi:10.1081/JBC-100102126
Blank M., Goodman R. (2008): A mechanism for stimulation of biosynthesis by electromagnetic fields: charge transfer in DNA and base pair separation. J. Cell Physiol. 214, 20-26; doi:10.1002/jcp. 21198

Cellini L., Grande R., Di Campli E., Di Bartolomeo S., Di Giulio M., Robuffo I., Trubiani O., Mariggiò M. A. (2008): Bacterial response to the exposure of $50 \mathrm{~Hz}$ electromagnetic fields. Bioelectromagnetics 29, 302-311; doi:10.1002/ bem. 20391

Chow K. C., Tung W. L. (2000): Magnetic field exposure enhances DNA repair through the induction of DNA K/J synthesis. FEBS Lett. 478, 133-136; doi:10.1016/S00145793(00)01822-6

Coult L. A, Harris, P. A., Barker A. T., Graham Pockley A. (2004): Effect of $50 \mathrm{~Hz}$ electromagnetic fields on the induction of heat-shock protein gene expression in human leukocytes. Radiat. Res. 161, 430-434; doi:10.1667/RR3145

Del Re B., Garoia F., Mesirca P., Agostini C., Bersani F., Giorgi G. (2003): Extremely low frequency magnetic fields affect transposition activity in Escherichia coli. Radiat. Environ. Biophys. 42, 113-118; doi:10.1007/s00411003-0192-9

Del Re B., Bersani F., Agostini C., Mesirca P., Giorgi G. (2004): Various effects on transposition activity and survival of Escherichia coli cells due to different ELF-MF signals. Radiat. Environ. Biophys. 43, 265-270; doi:10.1007/ s00411-004-0260-9

Del Re B., Bersani F., Mesirca P., Giorgi G. (2006): Synthesis of DnaK and GroEL in Escherichia coli cells exposed to different magnetic field signals. Bioelectrochemistry 69, 99-103; doi:10.1016/j.bioelechem.2005.11.006

Di Carlo A. L., White N. C., Litovitz T. A. (2001): Mechanical and electromagnetic induction of protection against oxidative stress. Bioelectrochemistry 53, 87-95; doi:10.1016/S03024598(00)00116-1

George I., Geddis M. S., Lill Z., Lin H., Gomez T., Blank M., Oz M. C., Goodman R. (2008): Myocardial function improved by electromagnetic field induction of stress protein hsp70. J. Cell Physiol. 216, 816-23; doi:10.1002/jcp.21461

Goodman R., Blank M. (1998): Magnetic field stress induces expression of hsp70. Cell Stress \& Chaperones 3, 79-88; doi:10.1379/1466-1268(1998)003<0079: MFSIEO $>2.3 . C O ; 2$

Guisbert E., Herman C., Lu C. Z., Gross C. A. (2004): A chaperone network controls the heat shock response in E. coli. Genes Dev. 18, 2812-2821; doi:10.1101/gad.1219204

Guisbert E. Yura T. Rhodius V. A., Gross C. A. (2008): Convergence of molecular, modeling, and systems approaches for an understanding of the Escherichia coli heat shock response. Microbiol. Mol. Biol. Rev. 72, 545-554; doi:10.1128/MMBR.00007-08

Gutzeit H. O. (2001): Biological effects of ELF-EMF enhanced stress response: new insights and new questions. Electromagn. Biol. Med. 20, 15-26; doi:10.1081/JBC-100103157

Henderson B. R., Pfister G., Boeck G., Kind M., Wick G. (2003): Expression levels of heat shock protein 60 in human endothelial cells in vitro are unaffected by exposure to $50 \mathrm{~Hz}$ magnetic fields. Cell Stress \& Chaperones 
8, 172-82; doi:10.1379/1466-1268(2003)008<0172: ELOHSP $>2.0 . \mathrm{CO} ; 2$

Henderson B., Kind M., Boeck G., Helmberg A., Wick G. (2006): Gene expression profiling of human endothelial cells exposed to $50 \mathrm{~Hz}$ magnetic fields fails to produce regulated candidate genes. Cell Stress \& Chaperones 11, 227-232; doi:10.1379/CSC-196.1

Huisman O., Kleckner N. (1987): A new generalizable test for detection of mutations affecting Tn10 transposition. Genetics 116, 185-189

Kroupova J., Bartova E., Foit L., Strasak L., Kozubek S., Vetterl V. (2007): Low-frequency magnetic field effect on cytoskeleton and chromatin. Bioelectrochem. 70, 96-100; doi:10.1016/j.bioelechem.2006.03.034

Lacy-Hulbert A., Metcalfe J. C., Hesketh R. (1998): Biological responses to electromagnetic fields. FASEB J. 12, 395-420

Nakasono S., Saiki H. (2000): Effect of ELF magnetic fields on protein synthesis in Escherichia coli K12. Radiat. Res. 154, 208-216; doi:10.1667/0033-7587(2000)154[0208: EOEMFO]2.0.CO;2

Novak J., Strasak L., Foit L., Slaninova I., Vetterl V. (2007): Effects of low-frequency magnetic fields on the viability of yeast Saccharomyces cerevisiae. Bioelectrochemistry 70, 115-121; doi:10.1016/j.bioelechem.2006.03.029

Smith O. (1996): Cells, stress and EMFs. Nature Med. 2, 23-24; doi:10.1038/nm0196-23

Twiss E., Coros A. B., Tavakoli N. P., Derbyshire K. M. (2005): Transposition is modulated by a diverse set of host factors in Escherichia coli and is stimulated by nutritional stress.
Molecular Microbiol. 57, 1593-1607; doi:10.1111/j.13652958.2005.04794.X

Ventura C., Maioli M., Asara Y., Santoni D., Mesirca P., Remondini D., Bersani F. (2005): Turning on stem cell cardiogenesis with extremely low frequency magnetic fields. FASEB J. $19,155-157$

Williams P. A., Ingebretsen R. J., Dawson R. J. (2006): 14.6 mT ELF magnetic field exposure yields no DNA breaks in model system Salmonella, but provides evidence of heat stress protection. Bioelectromagnetics 27, 445-450; doi:10.1002/bem. 20210

Winter J., Linke K., Jatzek A., Jakob U. (2005): Severe oxidative stress causes inactivation of DnaK and activation of the redox-regulated chaperone Hsp33. Mol. Cell. 17, 381-92; doi:10.1016/j.molcel.2004.12.027

Wolf F. I., Torsello A., Tedesco B., Fasanella S., Boninsegna A., D’Ascenzo M., Grassi C., Azzena G. B., Cittadini A. (2005): $50 \mathrm{~Hz}$ extremely low frequency electromagnetic fields enhance cell proliferation and DNA damage: possible involvement of redox mechanism. Biochim. Biophys. Acta 1743, 120-129; doi:10.1016/ j.bbamcr.2004.09.005

Yura T., Kanemori M., Morita M. T. (2000): The heat shock response: regulation and function. In: Bacterial Stress Responses. (Eds. G. Storz and R. Hengge-Aronis), pp 3-18, ASM Press, Washington, DC

Received: July 17, 2009

Final version accepted: October 7, 2009 